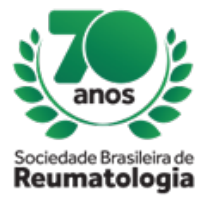

\title{
PLANT BASED PLATFORMS FOR IMMUNOBIOLOGICALS PRODUCTION
}

Luiz Eduardo Azevedo Ramos Silva (IPEMED, São Paulo, SP, Brasil), Ana Paula Bazilio (IPEMED, São Paulo, SP, Brasil), Maria Goreti Rosa Freitas (FIOCRUZ, Rio de Janeiro, RJ, Brasil), Doug Coussar (PlantForm Company., Canadá), Donald I. H. Stewart (PlantForm Company., Canadá), Germana Regazzi (PlantForm do brasil Biotecnologia Ltda., Rio de Janeiro, RJ, Brasil)

\section{BACKGROUND}

Prokaryotic and eukaryotic organisms share the same basic biochemical pathways for protein production, denoting their common evolution. The use of prokaryotic cells as bioreactors coincides with the advent of agriculture in human history and dates back to 10,000 years ago with fermentation for the production of wines, cheeses and breads.

However, the production of pharmacologically active drugs began in the decade 1970 s with the use of Escherichia coli bacterial cells for production of a recombinant Insulin (Daniel C. et al.,1982).

The use of animal eukaryotic cells was initiated with cell cultures of Chinese Hamster Ovary Cells - CHO, a breakthrough in pharmaceutical biotechnology which dates from 30 years ago (Wei-Shou Hu, et al, 2007). The use of $\mathrm{CHO}$ cells allowed the expression and manufacture of complex, high molecular weight molecules such as antibodies and complex proteins.

A little later, at 1989 (Hiatt et al.,1989), plant eukaryotic cells came to join the arsenal of pharmaceutical biotech, with many advantages: 1 Safety, because no human pathogens replicate in plants (Commandeur et al.,2003); 2 Simple structure, because there is no need for a sterile environment (Sack et al., 2015); 3 Low cost, as opposed to the expensive media required for mammalian cell cultures (Xu et al.,2016); and 4 Fast production, that can be obtained in 8 weeks after receiving the corresponding DNA (Shoji et al,2012).

Immunobiologicals commonly used in rheumatology such as adalimumab, rituximab, infliximab, golimumab and others used for rheumatoid arthritis, psoriatic arthritis and ankylosing spondylitis are being produced by means of plant based systems (Buyel 2018. doi: 10.3389/fpls.2018.01893).

\section{MATERIALS AND METHODS}

Comparison of current pharmacologically active drug production platforms and worldwide licensed drugs were carried out by bibliographical search in PubMed, Google Scholar and CrossRef.

\section{RESULTS}

Drug production by plant-based expression showed differences in terms of cost, purity and time of production compared to conventional cell culture.

\section{CONCLUSION}

Drug production plant platforms shows the superiority of plant platforms in terms of cost, purity and safety. Plant-based platforms should be the biotechnology methodology of choice for both economic and technical-scientific reasons including low initial investment, ease of installation and sustainability. 